\title{
Filter Coefficient Selection Using Design Criteria
}

\author{
John E. Gray and George J. Foster \\ Code N92 \\ Naval Surface Warfare Center Dahlgren Division \\ Dahlgren, VA. 22448 \\ jgray@relay.nswc.navy.mil \\ gfoster@relay.nswc.navy.mil
}

\begin{abstract}
For tracking systems with a uniform data rate and stationary measurement noise, non-maneuvering targets can be accurately tracked with a steady-state Kalman filter. The steady-state Kalman filter, which can be viewed as equivalent to an alpha-beta filter, has been widely applied to many different systems. A means of selecting the filter coefficients was proposed by Kalata using Kalman filter considerations. An alternative method based on noise reduction ratios is presented in this paper. Using a design criteria with the Kalata relation, optimal filter coefficients can be selected for specific applications. This method generalizes current methods for selecting the filter coefficients.
\end{abstract}

\section{INTRODUCTION}

With a tracking radar, it is possible to measure the position of the target directly, but it is not possible to measure the velocity of the target directly. A means of estimating the future positions and velocities of the target is needed. Some of the earliest filters used in tracking radars are the $\alpha-\beta$ filter and the $\alpha-\beta-\gamma$ filter [1]. The variable gain $\alpha-\beta$ filter combines elements of the Kalman filter [2] [3]. The $\alpha-\beta$ filter has found application when large numbers of objects are to be tracked. The tracking equations for the $\alpha-\beta$ filter consists of two parts: prediction equations, which are given by

$$
\begin{gathered}
x_{p}(k)=x_{s}(k-1)+v_{s}(k-1) T \\
v_{p}(k)=v_{s}(k-1)
\end{gathered}
$$

and smoothing equations

$$
x_{s}(k)=x_{p}(k)+\alpha\left(x_{m}(k)-x_{p}(k)\right)
$$

$$
v_{s}(k)=v_{s}(k-1)+\frac{\beta}{T}\left(x_{m}(k)-x_{p}(k)\right)
$$

where

- $x_{s}(k)$ smoothed position at the k-th interval

- $x_{p}(k)$ predicted position at the $\mathrm{k}$-th interval

- $x_{m}(k)$ measured position at the $\mathrm{k}$-th interval

- $v_{s}(k)$ smoothed velocity at the $\mathrm{k}$-th interval

- $v_{p}(k)$ predicted velocity at the $\mathrm{k}$-th interval

- $T$ radar update interval or period

- $\alpha, \beta$ filter weighing coefficients

These equations are one-dimensional, but can be extended to three dimensions by substituting successively $y$ and $z$ for $x$ in Eqs. (1-4). The filter equations are usually analyzed in one dimension and the resulting analysis is extended to three dimensions with the assumption that this gives similar results.

The first major advance in using the tracking equations was to find a means to optimally select a value for $\beta$ in terms of $\alpha$. The functional relationship between $\beta$ and $\alpha$ was derived by [4] using a combination of $Z$-transforms and variational calculus. Benedict derived an optimal relationship between the coefficients $\alpha$ and $\beta$, the so called BenedictBordner relation

$$
\beta_{B B}=\frac{\alpha^{2}}{2-\alpha}
$$

This relationship is optimal in the sense that it is a compromise between the twin goals of good noise reduction which requires small $\alpha$ (and thus, small 
$\beta)$, and good tracking through maneuvers which requires a larger $\beta$. The noise reduction ratio for the smoothed position is given by

$$
K_{x}(0)=\frac{2 \alpha^{2}+\beta(2-3 \alpha)}{\alpha(4-2 \alpha-\beta)}
$$

while the noise reduction for the smoothed velocity is

$$
K_{v}(0)=\frac{2 \beta^{2}}{T^{2}} \frac{1}{\alpha(4-2 \alpha-\beta)}
$$

Thus with measurement noise with variance $\sigma_{n}$, the output of the filter, for the smoothed velocity for example, has a reduced variance $\sqrt{K_{v}(0)} \sigma_{n}$.

A means for selecting $\alpha$ was introduced by Kalata [5] who defined a variable $\Gamma$, known as the tracking index. The tracking index is a function of the assumed target maneuverability variance $\sigma_{w}^{2}$ (deviation from modeled behavior or the process noise in a Kalman filter) and the measurement noise variance $\sigma_{n}^{2}$, e.g.

$$
\Gamma_{K}=\frac{\sigma_{v} T^{2}}{\sigma_{w}}=\frac{\beta}{\sqrt{1-\alpha}}
$$

In addition, he suggested using the relationship between $\alpha$ and $\beta$

$$
\beta_{K}=2(2-\alpha)-4 \sqrt{1-\alpha}
$$

which is more useful in steady state conditions. Note the Kalata and Benedict-Bordner two relationships are numerically equivalent for $\alpha<.4$.

Note by following the Kalata relationships, it is possible to implement an $\alpha-\beta$ filter that has nearly identical performance characteristics to a two state Kalman filter. While there is no great advantage to this for a simple tracking system that is tracking fewer than ten objects at a time, there is considerable advantage to the $\alpha-\beta$ filter implementation of a Kalman filter in a rich tracking environment for real time implementation. With this said, there are alternative principles that can be introduced into the design of an $\alpha-\beta$ filter so that it is no longer entirely equivalent to a steady state Kalman filter.

The process noise is an edifice that was constructed in order to obtain solutions to the gain equations. It reflects an unknown, namely the noise associated with target motion, rather than the measurement of the target motion which is measured from the sensor. It reflects a decomposition of a noise process into two components, sensor measurement noise and target motion noise. Under some circumstances such an artificial de-construction might be useful as in the case of air-to-air radars. It is somewhat artificial in nature, and thus less adaptive than other criteria that can be introduced in order to accomplish a tracking goal. Since the development of the Benedict-Bordner relation and the Kalman filter were almost concurrent, the ideas used by Benedict to arrive at his relations were largely ignored since apparently better results could be obtained from the Kalman filter. Thus, the possibility of using a design principle that replaces the process noise as a selection criteria for the gains was ignored because of the introduction of the Kalman filter's process noise models. We propose the introduction of different criteria to replace the process noise model as a means of increasing the generality of the Kalman filter structure to deal with a diverse environment of threats while at the same time maintaining the other aspects of the Kalman filter.

\section{Introduction of Cost Functions as a Design Criteria for Alpha Beta FILTERS}

The response of an $\alpha-\beta$ filter to a linear acceleration produces the model input produces three terms; a transient term, a lag, and the model input term [6]. The lag is a bias, so the expected value $E$ of the steady state response $E\left[x_{s}(k)-x_{m}(k)\right]$ is the lag. Similarly, $E\left[\left(x_{s}(k)-x_{m}(k)\right)^{2}\right]$ is equal to $K_{x}(0) \sigma_{n}^{2}+\frac{1}{2} L_{x}^{2} a_{0}^{2} T^{4}$ and $E\left[\left(v_{s}(k)-x_{m}(k)\right)^{2}\right]=$ $K_{v}(0) \sigma_{n}^{2}+L_{v}^{2} a_{0}^{2} T^{2}$. Note that this is based on the assumption that the noise is zero mean and has a variance $\sigma_{n}^{2}$. This allows one to define a cost or objective function for the smoothed velocity

$$
J_{V}(\alpha, \beta)=\sigma_{n}^{2} K_{v}(0)+\left(\frac{\alpha}{\beta}-\frac{1}{2}\right)^{2} a_{0}^{2} T^{2}
$$


This can be written in terms of $r(r=\sqrt{1-\alpha})$ using the Kalata relationship as

$$
J_{V}(r)=\frac{2 a^{2}(1-r)^{3}}{r(1+r)}+b^{2} \frac{r^{2}}{(1-r)^{2}}
$$

or

$$
C^{v}(u, a, \Gamma)=\frac{J_{V}(u)}{a^{2}}=\frac{2}{u(u+1)(1+2 u)}+\Gamma^{2} u^{2}
$$

where $a=\frac{\sigma_{n}}{T}, u=\frac{r}{(1-r)}, \Gamma=\frac{a_{0} T^{2}}{\sigma_{n}^{2}}$, and $b=a_{0} T$ This cost function can be minimized with respect to $u$, which gives the optimal value $u_{*}$

$$
\frac{1+6 u_{*}+6 u_{*}^{2}}{u_{*}\left(u_{*}+1\right)^{2}\left(1+2 u_{*}\right)^{2}}=\Gamma_{V}^{2} u_{*}^{2}
$$

This function can be solved for $\Gamma_{V}\left(u_{*}\right)$ by numerical methods. Note this $\Gamma_{V}$ is a pseudo tracking index. An alternative cost function based on the noise reduction ratio and the position lag is

$$
\frac{J_{p}}{\sigma_{n}^{2}}=\frac{(1-r)(1+2 r)}{(1+r)}+\frac{\Gamma^{2}}{8}\left(\frac{r}{1-r}\right)^{4}
$$

or

$$
C^{p}\left(u, \sigma_{n}, \Gamma\right)=\frac{(1+3 u)}{(1+2 u)(u+1)}+\frac{\Gamma^{2}}{8} u^{4}
$$

Minimizing the cost function with respect to $u$ gives

$$
\frac{2+3 u_{*}}{\left(1+u_{*}\right)^{2}\left(1+2 u_{*}\right)^{2}}=\frac{\Gamma_{P}^{2}}{4} u_{*}^{2},
$$

where $\Gamma_{P}^{2}$ is the same as above. Numerical methods can be used to determine $u_{*}=u\left(\Gamma_{P}\right)$.

To compare the results of using different criteria for selecting $\alpha$, one can, of course, use simulations to study the details of what is happening during the entire tracking process. Bounds on the performance can be obtained by examining the cost functions as a function of $\alpha$. The normalized cost functions for the velocities, which are evaluated in terms of their respective tracking indices $\Gamma(u)$, is for the Kalata relationship $C^{v}\left(u, a=1, \Gamma_{K}\right)=C_{K}^{v}$

$$
C_{K}^{v}=\frac{2}{u(u+1)(1+2 u)}+\frac{4}{(u+1)^{2}}
$$

while the velocity optimized relationship is $C^{v}\left(u, a=1, \Gamma_{V}\right)=C_{V}^{v}$

$$
C_{V}^{v}=\frac{2}{u(u+1)(1+2 u)}+\frac{1+6 u+6 u^{2}}{u(u+1)^{2}(1+2 u)^{2}}
$$

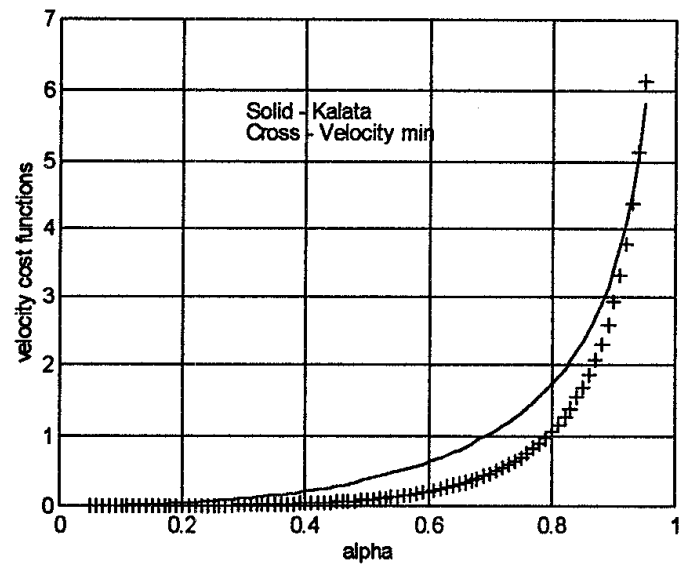

Figure 1 - Velocity Cost Functions

A graphical comparison of the two velocity cost functions in Figure I shows that $C_{V}<C_{K}$ for $\alpha$ in the range $0 \leq \alpha \leq .95$ which indicates a significant improvement in reduction in cost over conventional selection methods. The normalized cost functions for the positions, which are evaluated in terms of their respective tracking indices $\Gamma(u)$, is for the Kalata relationship $C^{p}\left(u, \sigma_{n}=1, \Gamma_{K}\right)=C_{K}^{p}$

$$
C_{K}^{p}=\frac{1+3 u}{(u+1)(1+2 u)}+\frac{u^{2}}{2(u+1)^{2}}
$$

while the position optimized relationship is $C^{p}\left(u, \sigma_{n} 1, \Gamma_{P}\right)=C_{P}^{p}$

$$
C_{P}^{p}=\frac{1+3 u}{(u+1)(1+2 u)}+\frac{(2+3 u) u^{2}}{2(u+1)^{2}(1+2 u)^{2}}
$$

A graphical comparison of the two pasition cost functions in Figure II shows that $C_{P}^{p}<C_{K}^{p}$ for $\alpha$ in the range $0 \leq \alpha \leq .95$ which indicates an improvement using the position minimization criteria over the conventional selection method.

An alternative to the previous criteria is to base the filter coefficient selection on the assumption that there is no plant noise, but instead a deterministic bias due to the target maneuvering. The output of the filter is the bias $L_{v} a_{0} T$, so the criteria to minimize is

$$
J=\sigma_{n} \sqrt{K_{v}}+L_{v} a_{0} T
$$




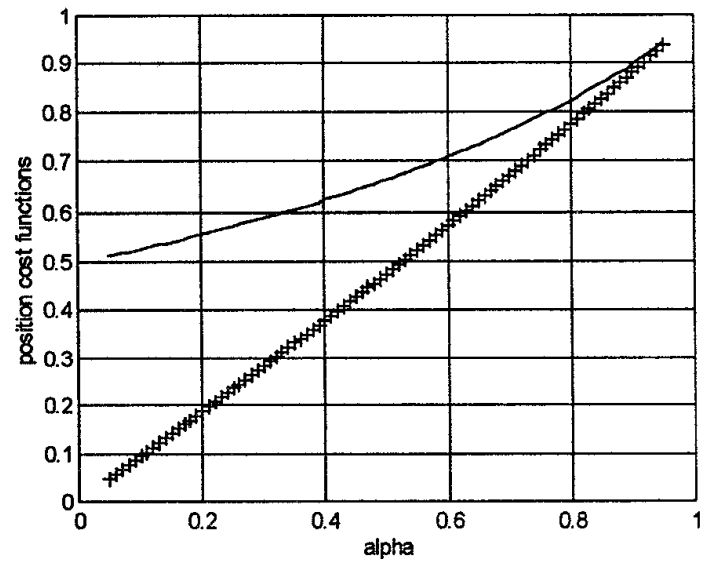

Figure 2 - Postion Cost Functions

or

$$
\frac{J(u)}{a}=\sqrt{\frac{2}{u(u+1)(1+2 u)}}+\Gamma u
$$

using the previous notation. Minimizing Eq. (21) for $u$ gives

$$
\Gamma=\frac{6 u^{2}+6 u+1}{(u(u+1)(1+2 u))^{3 / 2}}
$$

which can be readily solved for $u=u_{*}(\Gamma)$, the optimal value, using root solvers in MATLAB. Then $\alpha$ is

$$
\alpha=\frac{2 u_{*}+1}{\left(1+u_{*}\right)^{2}} .
$$

which is the optimal one for the criteria in Eq. (22). A direct comparison between this criteria and the others is not possible since they are based on different assumptions, however the $\alpha$ obtained by using Eq. (24) is virtually identical to the one obtained using Eq. (12). This suggests that using either one of these is preferable when a bias (deterministic or random) is present.

Different cost functions other than the ones above are useful under some circumstances. If there is concern about transient effects that occur while the target is accelerating replacing the lags by twice their value includes this effect since the transient response is always less than or equal to the lag. Another possibility is to weight the lag by the percentage of time maneuvers are expected to occur. In some cases, it is more desirable to minimize the lag in predicted response, so the cost functions of position and velocity are combined and then minimized.

\section{EXAMPLES}

One frequently hears that the $\alpha-\beta$ filter is too primitive to use in this time of fast computers and the Kalman filter. Strictly speaking, this is an example of cookbook thinking. In situations where the noise is range dependent, the Kalman filter gains always converge to steady state and then change slowly as the range of the object being tracked changes. Thus with proper initialization of the data, the $\alpha-\beta$ filter with a look up table of the coefficients as a function of range achieves the same performance as a Kalman filter. Unless one is tracking only a few objects, the advantage of the $\alpha-\beta$ filter implementation of the Kalman filter is to be preferred over the conventional Kalman filter for real time applications. It does require some care in the design in terms of understanding the operating environment as well as careful thought in the underlying design concepts.

With that understanding, we now consider several specific examples. When tracking is done in Cartesian coordinates, the noise is range dependent. Typically, the noise can be written as $\sigma_{x}=R \sigma_{\theta}$, where $\sigma_{\theta}$ is the sensor angular noise, which is a known parameter of the tracking system. Given that the range is in kilometers and the angle noise is in milliradians, the noise can be written as a function of the range $(n)$ times a constant $k$. The tracking index becomes

$$
\Gamma=\frac{a_{0} T^{2}}{k n} .
$$

Specific system parameters are then plugged into Eq. (25) and the $\alpha^{\prime} s$ are computed. For example, commercial aircraft do not have maneuvers that exceed $3 g^{\prime} s$. We choose other system parameters so that the tracking index is $\Gamma=\frac{1.76}{n}$. The table shows the two different $\alpha^{\prime} s$ computed from the common tracking index and the resultant costs for ranges from $4-128 \mathrm{~km}$.

Two other examples of interest to the tracking community can be mapped into the same form as the accelerating target with different interpretations of the coefficients used to form the tracking index. A maneuvering target that has a turning rate of less than $15^{\circ} \mathrm{deg} / \mathrm{sec}$ has an acceleration that is equivalent to a constant acceleration so the $b$ in Eq. (12) can be represented by $\rho_{0} \omega^{2}$ where $\omega$ is the turning rate and $\rho_{0}$ is the radius of curvature of the turn. 


\begin{tabular}{|c||c||c||c||c||c|}
\hline Range $(n)$ & $\Gamma$ & $\alpha_{K}$ & $\alpha_{R}$ & $C_{K}$ & $C_{R}$ \\
\hline 4 & .69 & .686 & .896 & .9678 & 1.5662 \\
8 & .345 & .5616 & .7783 & .5312 & .4983 \\
16 & .1725 & .4449 & .6537 & .2786 & .2100 \\
32 & .0863 & .3395 & .5324 & .1492 & .0897 \\
64 & .0431 & .2542 & .4274 & .0776 & .0396 \\
128 & .0216 & .1876 & .3392 & .0401 & .0177 \\
\hline
\end{tabular}

the optimal coefficients for this specific cost $J(\alpha, \beta)$.

Different cost functions lead to selection criteria depending on one's application. Thus, a more flexible criteria can be introduced into a "Kalman filter" that allows one to simultaneously track more than one type of target class. Note, this idea has been forshadowed to some extent in a more general context

Table 1. Cost Functions vrs Range

The noise is now angular $\sigma_{n} \Rightarrow R \sigma_{\theta}$ and to a $98 \%$ confidence level, $a$ becomes $3 \sigma_{\theta} R / T$. Thus this example is completely mapped into the solution in Eq. (12) with

$$
\Gamma_{m}=\frac{\rho_{0} \omega^{2} T^{2}}{3 R \sigma_{\theta}} .
$$

Another example is to determine the ballistic coefficient of an object. The force on an object undergoing a ballistic slowdown is $F=\frac{1}{2} \rho k v^{2}$. This force produces an equivalent acceleration term $a_{0}=$ $\rho \frac{\left(\frac{k}{m}\right)^{2}}{v_{0}^{2}}$, which can be used to give the tracking index

$$
\Gamma_{m}=\frac{\rho k^{2} T^{2}}{3 m^{2} v_{0}^{2} R \sigma_{\theta}}
$$

\section{Summary and Conclusions}

Some discussion is need to clarify some points. The tracking indexes discussed in this section are based on both deterministic and random components, while the Kalata tracking index is based on two random components. The Kalata tracking index has an acceleration term that is not observable, so it is at best an empirical quantity. While the minimization criteria uses the deterministic acceleration as a design criteria and hence it is not directly applicable to a particular tracking example, it is applicable to a class of tracking problems. Namely, if one wants to choose the "best" $\alpha$, in the sense of minimizing the cost function, for an upper limit on threat accelerations and sensor noise, the velocity cost function selection criteria gives better performance than the Kalata selection criteria. Thus it should be viewed as an alternative to the standard Kalman filter with a cost function or selection criteria replacing the plant noise model.

It is now apparent that the selection of the filter coefficients can be viewed as a process independent of the Kalman filter selection criteria, namely the process noise. In place of introducing process noise, a specified criteria is minimized to determine in the literature in article by Swerling [7] that suggests a great deal of ground remains to be explored along these lines.

\section{A. ACKNOWLEDGMENTS}

Thanks to Frank Reifler of Martin Marietta (Morestown) and William Murray (NSWC) for useful discussion of this and other aspects of filter design over the years.

\section{REFERENCES}

[1] S. S. Blackman, Multiple Target Tracking with Radar Applications, Artech House, 1986.

[2] A. Gelb, Applied Optimal Estimation, Cambridge, MA, MIT Press, 1974.

[3] Y. Bar-Shalom and T. Fortmann, Tracking and Data Association, Academic Press, Inc., Orlando, FL (1988).

[4] T. Bendict and G. Bordner, "Synthesis of an Optimal Set of Radar Track-While Scan Smoothing Equations", IRE Trans. on Automatic Control, AC-7, pp. 27-32, 1962.

[5] P.R. Kalata, "The Tracking Index: A Generalized Parameter for alpha-beta and alpha-betagamma Target Trackers," IEEE Trans. Aero. Elect. Syst., Vol AES-20, No. 2, pp. 174-182, March 1984.

[6] J.E. Gray and W.J. Murray, "The Response of the Transfer Functions of the Alpha-Beta Filter to Various Measurement Models," In Proceedings of the 29rd Southeastern Symposium on Systems Theory, pp. 389-393, Columbia, SC, March 1991.

[7] P. Swerling, "Modern State Estimation Methods from the Viewpoint of the Method of Least Squares", IEEE Trans. on Automatic Control, Vol. AC-16, No. 6, December 1971. 